\title{
Relativistic Pure Rotational Energy for Diatomic Molecules
}

\author{
Nicola Aladrah ${ }^{1}$, Adel Almoukhalalati ${ }^{1}$, and Suleiman Dibo ${ }^{1}$ \\ ${ }^{1} \mathrm{Al}$ Baath University
}

January 24, 2022

\begin{abstract}
This paper provides a simplified solution of the Dirac equation for the pure rotational energy of the diatomic molecules and a discussion of the non-relativistic limit. The last works [1-2] led to a complicated form of the relativistic energy of the molecular rotation-vibrational energy with high computational cost based on Schrodinger-like equation. The present work provides a way to determine the pure rotational energy without exclusion of the wavefunction components or using the Schrodinger-like equation, where the selection rule $\Delta J= \pm 1$, where $J$ is the rotational quantum number, appears as a prerequisite for solving the equations. Based on the anti-hermitian spin-orbit operator $(\mathrm{L})$ that been introduced in this paper, which excludes the derivatives, the computational cost expects to decrease.
\end{abstract}

\section{Hosted file}

Relativistic Pure Rotational Energy for Diatomic Molecules.docx available at https: //authorea.com/users/455088/articles/553894-relativistic-pure-rotational-energy-fordiatomic-molecules 